\title{
Some Infrared Bands of Deuterium Sulfide
}

\author{
Harry C. Allen, Jr., Earle K. Plyler, and L. R. Blaine
}

\begin{abstract}
Two regions of the $\mathrm{D}_{2} \mathrm{~S}$ spectrum have been observed, 5 and $3.6 \mu$. An analysis of the absorption in the $3.6 \mu$ region attributes it to two overlapping bands with excited states $\left(v_{1}, v_{2}, v_{3}\right)=(1,1,0)$ and $(0,1,1)$. The band center of the $(0,1,1)$ band is found to be some $50 \mathrm{~cm}^{-1}$ lower than the previously accepted value while the $(1,1,0)$ band center is found some $60 \mathrm{~cm}^{-1}$ higher than the previous determination. The absorption near $5 \mu$ seems to arise from the $(0,0,1)$ band centered near $1906 \mathrm{~cm}^{-1}$. Overlapping atmospheric absorption makes a detailed analysis impossible at this time. This band center is almost $100 \mathrm{~cm}^{-1}$ lower than previously reported. The excited state constants for the two bands near $3.6 \mu$ are
\end{abstract}

$$
\begin{array}{llll}
(1,1,0) \nu_{0}=2742.77 & A^{*}=5.478 & B^{*}=4.512 & C^{*}=2.395 \\
(0,1,1) \nu_{0}=2754.44 & A^{*}=5.531 & B^{*}=4.521 & C^{*}=2.405 .
\end{array}
$$

\section{Introduction}

Although the spectrum of hydrogen sulfide is now fairly well understood, very little work has been done on the totally deuterated compound. The spectrum of $\mathrm{D}_{2} \mathrm{~S}$ was investigated with low resolution by Bailey, Thompson, and Hale [1] ${ }^{2}$ and with somewhat higher resolution by Nielsen and Nielsen [2]. In general these two groups agreed as to the regions of absorption, the only real discrepancy arising in the region near $3.6 \mu$. Although Nielsen and Nielsen resolved the rotational structure in several regions they did not present a rotational analysis of any of their data. The vibrational assignments of the two groups were completely different. With this considerable confusion existing it was thought advisable to reinvestigate the spectrum of $\mathrm{D}_{2} \mathrm{~S}$.

One $\mathrm{D}_{2} \mathrm{~S}$ band has been successfully analyzed [3] previously, and in the present work the analysis of the rotational structure in the region of $2750 \mathrm{~cm}^{-1}$ is reported. The fundamental region near $1900 \mathrm{~cm}^{-1}$ was also investigated although the atmospheric absorption in this region makes a complete rotational analysis quite impossible at this time.

\section{Experimental Procedure}

The intensity of absorption of $\mathrm{D}_{2} \mathrm{~S}$ is weak, thus it was necessary to use several centimeters pressure of the gas and a path length of $10 \mathrm{~m}$ to observe the absorption in the $3.6 \mu$ region. The absorption at $5 \mu$ could be observed satisfactorily with a $1 \mathrm{~m}$ cell and several centimeters pressure of the gas.

The spectra were recorded with the grating instruments of the Radiometry Section, using a 7,500 lines/in. grating with a ruled surface of about 6 by 8 in. and a cooled $\mathrm{PbTe}$ cell as a detector. The resolution was about $0.2 \mathrm{~cm}^{-1}$ The wavelengths of the lines were measured by employing a Fabry-Perot interferometer as previously described [4].

${ }^{1}$ The work reported herein was supported by the U. S. Atomic Energy Commission.

Figures in brackets indicate the literature references at the end of this paper.

\section{Analysis}

Figure 1 is a reproduction of a recorder trace of the absorption near $2750 \mathrm{~cm}^{-1}$. The absorption shows a well-defined $P_{-}, Q_{-}, R$-branch type of structure. The appearance of a collected $Q$-branch indicates that the change in electric moment is along the least inertial axis, hence $A$-type selection rules must be invoked [5].

Assignments of the strong lines in the $P$ - and $R$-branches of the band could be made by using the previously determined ground state $[3]$ of $\mathrm{D}_{2} \mathrm{~S}$. From these assignments estimates were made of the excited state inertial parameters from which a trial spectrum was calculated using published tables of the reduced energy [6], $E(\mathrm{~K})$.

Relative intensities of the transitions were calculated by combining the Boltzman factor and appropriate nuclear spin statistics with the published values of the line strength [5]. Line strength values were used for $K=0.5$. These were found to be close enough for our purposes. This trial spectrum was compared with the observed spectrum and adjustments were made to improve the agreement by means of the partial derivatives of the energy with respect to the various inertial constants [3]. The final adjustment of the parameters was obtained by carrying out a least squares treatment [3] in which some 90 assignments were used. The agreement between the observed absorption and calculated transitions for these 90 assignments was excellent. The average deviation between the observed and calculated values was $0.07 \mathrm{~cm}^{-1}$.

After assigning the strong transitions of the $A$-type band, it was found that there was a sizable number of strong absorption peaks still unaccounted for. Closer examination revealed that these peaks arose from a second transition obeying the selection rules for a $B$-type band. Proceeding in the same manner as for the $A$-type band it was possible to assign unambiguously about 70 transitions to these absorption peaks with an average deviation of 0.06 $\mathrm{cm}^{-1}$. Strong transitions of these two bands account for almost all the absorption in this region. 


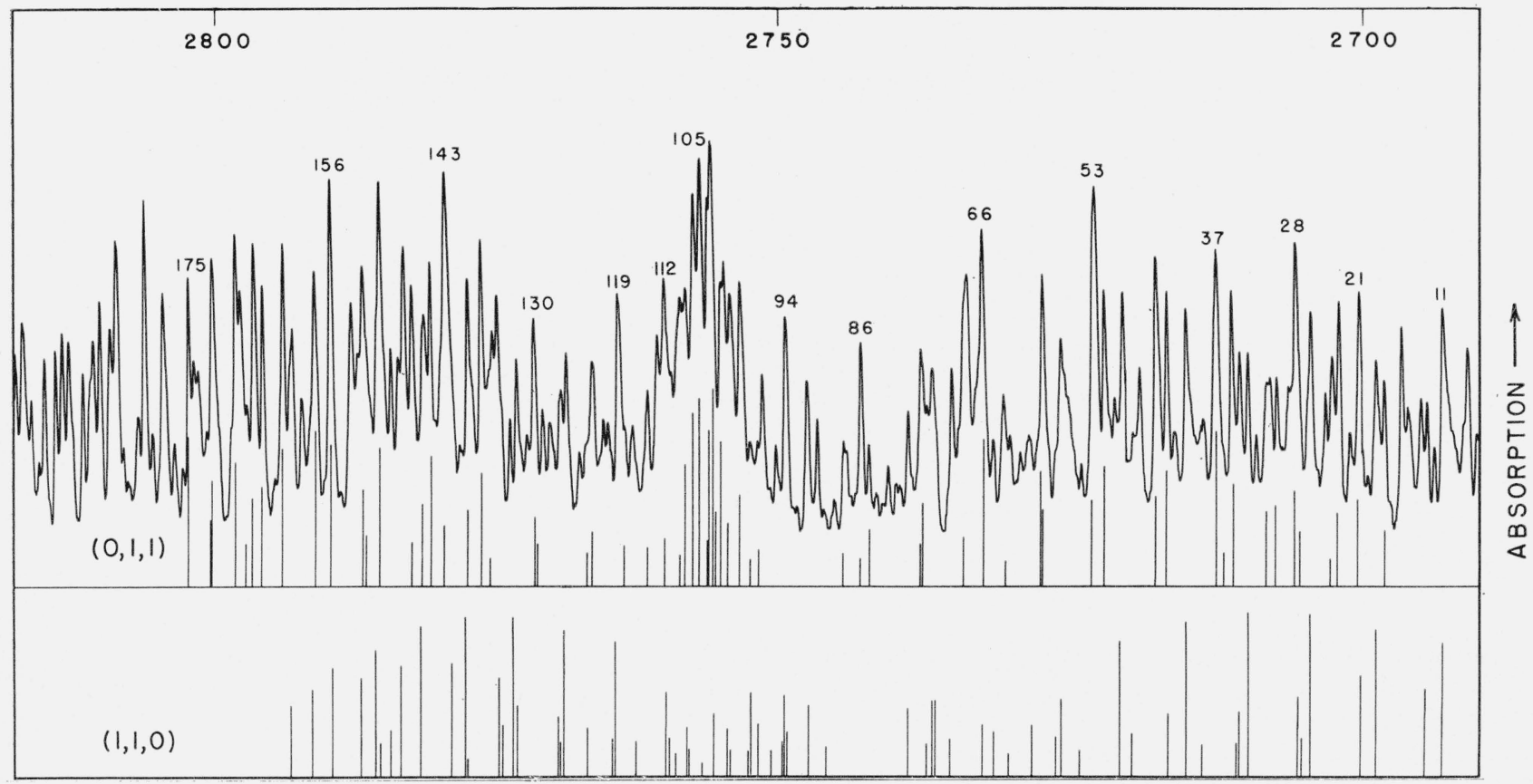

Figure 1. Comparison of observed and calculated spectrum of $\mathrm{D}_{2} \mathrm{~S}$ near $3.6 \mu$. The numbers on the peaks correspond to the numbers in column 1 of table 1.

The remaining peaks are due to a piling up of weaker transitions or to absorption by HDS which was present to a few percent in the sample. Because the moments of inertia in $\mathrm{D}_{2} \mathrm{~S}$ are relatively large, the resulting line spacing in the vibrational rotational bands is quite close, resulting in severe overlapping of the absorptions due to neighboring transitions both in the same band and in two closely lying bands. This situation leads to some difficulty in making an analysis for it cuts down the number of assignments that can be made, thus introducing more uncertainty into the determination of the inertial constants than one would like. The strongest transitions in the $P$ - and $R$-branches of both bands arise from levels that are strongly dependent on $C^{*}$. These levels could be readily identified and assigned, hence the value of $C^{*}$ for each band should be verv good. The strong Q-branch transitions of the $\dot{A}$ type band arise from levels strongly dependent on $A^{*}$. Although the calculated Q-branch reproduces the observed absorption in remarkable detail, indicating a good value for $A^{*}$, this may be somewhat fortuitous because many transitions of the $B$-type band are calculated to fall in this same region. The transitions determining $B^{*}$ most directly in both bands as well as $A^{*}$ in the $B$-type band are spread throughout the band and in general are weaker transitions. As a result these inertial constants are probably somewhat more uncertain than the others although the general excellence of the fit for the transitions in the two bands that could be unambiguously assigned indicates that the constants determined should be fairly reliable.
Figure 1 compares the observed absorption with the two calculated bands whereas table 1 compares the observed and calculated frequencies of some of the stronger transitions.

The excited state constants leading to the fit shown are

$$
\begin{array}{ccc}
A \text {-type } & \nu_{0}=2754.44 \mathrm{~cm}^{-1} \quad A^{*}=5.531 & B^{*}=4.521 \\
& C^{*}=2.403 & \\
B \text {-type } & \nu_{0}=2742.77 \mathrm{~cm}^{-1} \quad A^{*}=5.478 & B^{*}=4.512 \\
C^{*}=2.395 . &
\end{array}
$$

The previously determined ground [3] state was found to be adequate for both bands.

The absorption in the $5 \mu$ region is too complicated by atmospheric absorption to enable a quantitative analysis. However, the $\mathrm{D}_{2} \mathrm{~S}$ absorption seems to have a Q-branch located near $1906 \mathrm{~cm}^{-1}$ indicating an $A$-type band. The main transitions of the $P$ and $R$-branches that are free from contamination by water vapor absorption indicate that the center of the band is $1906 \pm 1 \mathrm{~cm}^{-1}$.

\section{Vibrational Asssignment}

The $A$-type band at $2754.44 \mathrm{~cm}^{-1}$ must have $v_{3}$ odd. The most likely transition with $v_{3}$ odd that could occur in this frequency region is the transition with the upper state $\left(v_{1}, v_{2}, v_{3}\right)=(0,1,1)$. The $B$-type band must have $v_{3}$ even and hence the most likely assignment on the basis of frequency is for the excited state to be $(1,1,0)$. 
TABLE 1. Comparison of observed and calculated spectra ${ }^{\text {a }}$

\begin{tabular}{|c|c|c|c|c|c|c|c|c|c|c|c|}
\hline \multirow{2}{*}{ Line } & \multirow{2}{*}{ Observed } & \multirow{2}{*}{ Transition } & \multicolumn{2}{|c|}{ Calculated } & \multirow{2}{*}{$\begin{array}{l}\text { Intensity } \\
\text { calculated }\end{array}$} & \multirow{2}{*}{ Line } & \multirow{2}{*}{ Observed } & \multirow{2}{*}{ Transition } & \multicolumn{2}{|c|}{ Calculated } & \multirow{2}{*}{$\begin{array}{l}\text { Intensity } \\
\text { calculated }\end{array}$} \\
\hline & & & $0,1,1$ & $1,1,0$ & & & & & $0,1,1$ & $1,1,0$ & \\
\hline & $\mathrm{cm}^{-1}$ & & $\mathrm{~cm}^{-1}$ & $\begin{array}{c}\mathrm{cm}^{-1} \\
2687.71\end{array}$ & 5.0 & & $\begin{array}{c}c m^{-1} \\
2732.68\end{array}$ & & $\mathrm{~cm}^{-1}$ & $\iota m^{-1}$ & \\
\hline 1 & 2687.79 & $\begin{array}{l}9-8-10-10 \\
9-9-10-9\end{array}$ & & 2687.71 & 2.5 & $\begin{array}{l}68 \\
69\end{array}$ & 2733.41 & & & & \\
\hline 2 & 2688.57 & $8-6-9-6$ & & 268920 & 39 & 70 & 2733. 68 & $2-30$ & 2733.86 & & 1.8 \\
\hline 3 & 2689.14 & $\begin{array}{l}8-6-9-6 \\
8-5-9-7\end{array}$ & & 2689.20 & 3.9 & $\begin{array}{l}71 \\
72\end{array}$ & $\begin{array}{l}2734.14 \\
2734.72\end{array}$ & $0_{0}-1_{0}$ & & 2734.85 & 1.9 \\
\hline $\begin{array}{l}4 \\
5\end{array}$ & $\begin{array}{l}2689.84 \\
2690.32\end{array}$ & & & & & $\begin{array}{l}73 \\
74\end{array}$ & $\begin{array}{l}2735.13 \\
2736.16\end{array}$ & $4_{0}-4_{2}$ & & 2736.20 & 4.6 \\
\hline 6 & 2690.54 & & & & & 75 & 2736.42 & $\left\{\begin{array}{l}3_{1}-3_{3} \\
n_{3}\end{array}\right.$ & & 2736.34 & 1.7 \\
\hline $\begin{array}{l}7 \\
8\end{array}$ & $\begin{array}{l}2691.40 \\
2691.74\end{array}$ & & & & & 76 & 2736.88 & $\begin{array}{c}2-2-20 \\
3-1-31\end{array}$ & & $\begin{array}{l}27356.44 \\
2736.72\end{array}$ & $\begin{array}{l}2.1 \\
1.9\end{array}$ \\
\hline $\begin{array}{r}9 \\
10\end{array}$ & $\begin{array}{l}2692.50 \\
2693.13\end{array}$ & & & & & $\begin{array}{l}77 \\
78\end{array}$ & $\begin{array}{l}2737.30 \\
2738.15\end{array}$ & $2-1-3-2$ & 2737.33 & & 4.3 \\
\hline 11 & 2693.38 & $8-8-9-8$ & & 2693. 38 & 5. 5 & 79 & $\begin{array}{l}2738.15 \\
2738.52\end{array}$ & $2_{0}-2_{2}$ & & 2738.48 & 3.7 \\
\hline 12 & 2694.12 & $8-7-9-9$ & & 2693.38 & 2.7 & $\begin{array}{l}80 \\
81\end{array}$ & $\begin{array}{l}2739.33 \\
2739.52\end{array}$ & $2-2-20$ & & 2739. 72 & 1.5 \\
\hline 13 & 2694.78 & $7-4-8-6$ & & 2694. 83 & 4. 1 & 82 & 2740.24 & & & & \\
\hline 14 & 2695. 22 & & & & & 84 & 2741.31 & $3-3-30$ & 2741.33 & & 1.1 \\
\hline $\begin{array}{l}15 \\
16\end{array}$ & $\begin{array}{l}2696.10 \\
2696.33\end{array}$ & & & & & 85 & 2741. 92 & $1-1-2-2$ & 2741. 91 & & 3.1 \\
\hline 17 & 2696.83 & & & & & 87 & 2743.94 & $I_{i j}=2-1$ & 2742.04 & & \\
\hline 18 & 2698.29 & $8-3-9-4$ & 2699. 22 & & 3. 0 & 88 & 2744.19 & $4-1-4_{0}$ & 2744.35 & & 1.8 \\
\hline 19 & 2698.95 & $\begin{array}{l}7-6-8-8 \\
7-7=8-7\end{array}$ & & $\begin{array}{l}2695.96 \\
2698.96\end{array}$ & $\begin{array}{l}5.8 \\
2.9\end{array}$ & $\begin{array}{l}89 \\
90\end{array}$ & $\begin{array}{l}2745.06 \\
2745.82\end{array}$ & $1_{1}-1-1$ & & 2745.83 & 1.5 \\
\hline 20 & 2699.78 & & & & & 91 & 2746. 44 & & & & \\
\hline 21 & 2700.44 & $\begin{array}{l}9-9-10_{-10} \\
9-8-10_{-9}\end{array}$ & $\begin{array}{l}2700.39 \\
2700.39\end{array}$ & & 4.6 & $\begin{array}{l}92 \\
93\end{array}$ & $\begin{array}{l}2747.33 \\
2748.34\end{array}$ & $2_{2}-2 \theta$ & & 27.47 .42 & 3.5 \\
\hline 22 & 2701. 22 & & 270213 & & & 94 & 2749.18 & $\left\{\begin{array}{l}3,-3-1 \\
a_{2}\end{array}\right.$ & & 2749. 05 & 1. 9 \\
\hline 23 & 2702.12 & $\begin{array}{l}8-5-y_{-6} \\
8_{-6}-9_{-7}\end{array}$ & $\begin{array}{l}2702.13 \\
2702.13\end{array}$ & & 3.9 & 95 & 2750.14 & $\mid 2 n-2-2$ & & 2749.23 & \\
\hline 24 & 2702.74 & $60-7-1$ & 2702.64 & & 1.5 & 96 & 2751.31 & & & & \\
\hline 25 & 2703. 33 & $6-6-7-6$ & & 2704.45 & 6. 1 & 97 & 2751.72 & $\left\{\begin{array}{l}4_{1}-4_{2} \\
4_{1}-4\end{array}\right.$ & 2751.59 & 2751.72 & $\begin{array}{l}3.3 \\
2.8\end{array}$ \\
\hline 26 & 2704.45 & $6-5-7-7$ & & 2704.45 & 3. 0 & 98 & 2752.38 & $1_{0}-1-1$ & 2752.32 & & 1.3 \\
\hline 27 & 2704.80 & $e_{1}^{6}-7_{0}$ & 2704.76 & & 2.9 & 99 & 2753. 24 & $\left\{\begin{array}{l}21-22 \\
2-5\end{array}\right.$ & $\begin{array}{l}2753.27 \\
2753.38\end{array}$ & & 4.? \\
\hline 28 & 2705.78 & $\begin{array}{l}8-7-9-8 \\
8-8-9-9\end{array}$ & $\begin{array}{l}2705.87 \\
2705.87\end{array}$ & & 5.3 & 100 & 2754.10 & $\begin{array}{l}(02-53 \\
2-1_{0}\end{array}$ & & 2754. 16 & 2.5 \\
\hline 29 & 2706.42 & & & & & 101 & 2754.56 & & & & 70 \\
\hline 30 & 2707. 38 & $\begin{array}{l}7-5-8-6 \\
7-4=8-5\end{array}$ & $\begin{array}{l}2707.38 \\
2707.38\end{array}$ & & 4. 4 & 102 & 2754.84 & $\begin{array}{l}43-44 \\
33_{3}-3_{2}\end{array}$ & $\begin{array}{l}2750.08 \\
2755.73\end{array}$ & & 6.7 \\
\hline 31 & 2707. 96 & $6-2-7-3$ & 2708.16 & & 1.8 & 103 & 2755.72 & $\begin{cases}0 & 4 \\
4 & -4 \\
5 & -5\end{cases}$ & $\begin{array}{l}2755.74 \\
2755.80\end{array}$ & & $\begin{array}{l}3.9 \\
40\end{array}$ \\
\hline 32 & 2708.26 & $\begin{array}{c}6-1-7-2 \\
5_{3}-6_{2}\end{array}$ & $\begin{array}{l}2708.37 \\
2708.42\end{array}$ & & $\begin{array}{l}3.6 \\
3.1\end{array}$ & & & $\mid \begin{array}{l}54-55 \\
64-6 ?\end{array}$ & & 2755.86 & \\
\hline 33 & 2703.13 & & & & & 104 & $2750 . .04$ & $\left\{\begin{array}{l}55-5 i \\
0\end{array}\right.$ & $\begin{array}{l}2756.06 \\
27560\end{array}$ & & 8.0 \\
\hline 34 & 2709.84 & $\begin{array}{l}5-4-6-6 \\
5-5-6-6\end{array}$ & & $\begin{array}{l}2709.84 \\
2709.84\end{array}$ & $\begin{array}{l}6.1 \\
3.1\end{array}$ & $100 t$ & & $\begin{array}{l}22_{2}-2 \\
\left\{1_{1}-10\right.\end{array}$ & $\begin{array}{l}2756.09 \\
2756.55\end{array}$ & & $\begin{array}{l}2.4 \\
2.3\end{array}$ \\
\hline 35 & 2710.54 & $\begin{array}{ll}-5 & -5 \\
4 & -5\end{array}$ & & $\begin{array}{l}2710.84 \\
2710.40\end{array}$ & $\begin{array}{l}0.1 \\
3.9\end{array}$ & 105 & 2756.71 & $\begin{cases}1 & 10 \\
6 & -65\end{cases}$ & 2756.63 & & 3.6 \\
\hline 36 & 2711. 22 & $\begin{array}{l}7-7-8-8 \\
7-6=8-7\end{array}$ & $\begin{array}{l}2711.29 \\
2711.29\end{array}$ & & 5.4 & 106 & 2757.28 & $\left\{\begin{array}{l}7_{6}-7 \\
7_{i}-7_{6}\end{array}\right.$ & $\begin{array}{l}2757.31 \\
2757.38\end{array}$ & & $\begin{array}{l}3.0 \\
6.0\end{array}$ \\
\hline & & $6_{-3}-7-4$ & 2712. 53 & & 4. 7 & 107 & 2758.03 & & & & \\
\hline 37 & 2712. 56 & $\begin{array}{l}6-4-7-5 \\
5-1-6-2\end{array}$ & $\begin{array}{l}2712.54 \\
2712.65\end{array}$ & & 3.0 & 108 & 2753.54 & $\left\{\begin{array}{l}60-3-2 \\
10-4\end{array}\right.$ & 2758.56 & 2758.50 & $\begin{array}{l}2.6 \\
1.7\end{array}$ \\
\hline $\begin{array}{l}38 \\
39\end{array}$ & $\begin{array}{l}2713.53 \\
2713.70\end{array}$ & $5_{0}-6-1$ & 2713.59 & & 1.8 & 109 & 2758.86 & $5-1-5-3$ & & $\begin{array}{l}2759.03 \\
0=50\end{array}$ & 1.2 \\
\hline $\begin{array}{l}39 \\
40\end{array}$ & 2714.10 & & & & & 110 & 2759.35 & $\begin{array}{l}63-61 \\
63-3\end{array}$ & & $\begin{array}{l}2,559.31 \\
2759.71\end{array}$ & $\begin{array}{l}1.2 \\
4.4\end{array}$ \\
\hline 41 & 2714.75 & $3-1-4-1$ & & 2714. 90 & 1.6 & 111 & $\begin{array}{l}2759.75 \\
2759.95\end{array}$ & $\left\{\begin{array}{l}3-2-2 \\
31-30\end{array}\right.$ & 2759.69 & & 2.6 \\
\hline 42 & 2715.08 & $\begin{array}{l}4-4-5-4 \\
4-2\end{array}$ & & $\begin{array}{l}2715.14 \\
2715.15\end{array}$ & $\begin{array}{l}5.8 \\
2.9\end{array}$ & & & $\mid 3-3-2-1$ & & 2759.78 & \\
\hline 43 & 2715.98 & $\begin{array}{r}4-3-3-5 \\
40-5-1\end{array}$ & 2716.16 & & 1.9 & 113 & 2760.56 & & & & \\
\hline 44 & 2716.63 & $6-6-7-7$ & 2716. 62 & & 6.2 & 114 & 2761.48 & $1-1-n_{0}$ & 2761.37 & 276162 & 2.0 \\
\hline 45 & 2717.40 & $0-5-7-6$ & 2716.62 & & & $\begin{array}{l}113 \\
116\end{array}$ & $\begin{array}{l}2762.60 \\
2762.60\end{array}$ & & & 20101.02 & \\
\hline 46 & 2717.58 & $5-3-6-4$ & 2718. 61 & & 4. 8 & 117 & 2762. 90 & & $2-c 3 c 3$ & & \\
\hline 47 & 2718.94 & & & & & 118 & 2763.46 & $\left\{\begin{array}{l}b_{1}-50 \\
4-4-3-2\end{array}\right.$ & 2653.53 & 2764.07 & 5.7 \\
\hline 48 & 2719.12 & $\begin{array}{l}20-30 \\
41-50\end{array}$ & 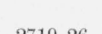 & 2719.07 & 2.6 & 119 & 2764.04 & $\{4-3-3-3$ & & 2764. 13 & 2.9 \\
\hline 49 & 2719.50 & & 2719. 26 & & & 121 & $\begin{array}{l}2764.90 \\
2765.36\end{array}$ & & & & \\
\hline 50 & 2720.36 & $\begin{array}{l}3-3-4-3 \\
3-4-4\end{array}$ & & $\begin{array}{l}2720.33 \\
2700.38\end{array}$ & 2.6 & 122 & 2766.34 & $2-1-10$ & 2766. 19 & & 2.9 \\
\hline 51 & 2721.06 & & & 2720.38 & 5.1 & $\begin{array}{l}125 \\
124\end{array}$ & $\begin{array}{l}2766.92 \\
2767.38\end{array}$ & $2-2-1-1$ & 2766.90 & & \\
\hline 52 & 2721.86 & $\begin{array}{l}5-5-6-6 \\
5-1-6-5\end{array}$ & $\begin{array}{l}2721.88 \\
2721.88\end{array}$ & & 6.3 & 125 & & $\int_{5}^{5-5}-4-3$ & & $\begin{array}{l}2768.56 \\
2768.60\end{array}$ & $\begin{array}{l}3.3 \\
6.6\end{array}$ \\
\hline 53 & 2722.70 & $4-1-5-2$ & 2722.77 & & 3. 0 & 120 & 2768.64 & $\left\{\begin{array}{l}0,-4-4-4 \\
4-2-3\end{array}\right.$ & & 2768.75 & $\begin{array}{l}0.0 \\
3.5\end{array}$ \\
\hline 54 & 2723.92 & $3_{1}-4_{0}$ & 2722. 94 & & 3.9 & 126 & 2769.14 & & & & \\
\hline 55 & $\begin{array}{l}2720.92 \\
2725,52\end{array}$ & $l_{1}^{1_{1}-2_{1}}$ & & $\begin{array}{l}2723.93 \\
2725.30\end{array}$ & $\begin{array}{l}\text { 1. } 3 \\
4.0\end{array}$ & $\begin{array}{l}127 \\
128\end{array}$ & $\begin{array}{l}2769.96 \\
2770.64\end{array}$ & & & & \\
\hline & & $2-1-3-3$ & & 2725.61 & $\begin{array}{l}1.0 \\
2.0\end{array}$ & 129 & 2771.38 & $3-2-2-1$ & 2771.27 & & 2.3 \\
\hline 56 & 2725.82 & $\begin{array}{r}33_{2}-4_{1} \\
5_{-3}-5\end{array}$ & 2725.61 & 2725.96 & $\begin{array}{l}1.2 \\
1.1\end{array}$ & $\begin{array}{l}130 \\
131\end{array}$ & $\begin{array}{l}2771.52 \\
2772.12\end{array}$ & & 2771.59 & & 4.7 \\
\hline 57 & 2726.79 & & & & & & & $3_{2}-2_{2}$ & & 2772. 91 & 3.7 \\
\hline 58 & 2727.11 & $\begin{array}{l}4-3-5-4 \\
4-4-5-5\end{array}$ & $\begin{array}{l}2727.07 \\
2727.07\end{array}$ & & 6.0 & 132 & 2772. 96 & $\left\{\begin{array}{l}6-5-5-5 \\
6-6-5-4\end{array}\right.$ & & $\begin{array}{l}2772.93 \\
2772.93\end{array}$ & $\begin{array}{l}3.6 \\
7.2\end{array}$ \\
\hline 59 & 2727.54 & $6-2-60$ & & 2727.55 & 2.7 & 133 & 2773.55 & & & & \\
\hline 61 & $\begin{array}{l}2728.48 \\
2728.45\end{array}$ & $53-5$ & & 2728,62 & 1.0 & 134 & 2774. 32 & $5-2-4-2$ & & 2774. 38 & 4. 6 \\
\hline 62 & 2729.01 & & & & 1.0 & $\begin{array}{l}135 \\
136\end{array}$ & $\begin{array}{l}2774.69 \\
2775.12\end{array}$ & & & & \\
\hline 63 & 2729.84 & $1-1-2-1$ & & 2729. 94 & 1.4 & 137 & 2775.49 & $3_{0}-2_{1}$ & 2775.44 & & 1.5 \\
\hline 64 & $\begin{array}{l}2730.28 \\
2731.24\end{array}$ & $\begin{array}{l}2_{2}-3_{1} \\
1\end{array}$ & 2730.52 & 2731.19 & $\begin{array}{l}1.5 \\
2.3\end{array}$ & 138 & 2776.06 & $\left\{\begin{array}{l}4-3-3-2 \\
4-4-3-3\end{array}\right.$ & $\begin{array}{l}2776.01 \\
2776.06\end{array}$ & & 6. 0 \\
\hline & & $2_{0}-3-1$ & 2732.16 & & 2.2 & 139 & 2776.93 & & & 277720 & 36 \\
\hline 66 & 2732.14 & $\left\{\begin{array}{l}3-3-4-4 \\
33-4-4\end{array}\right.$ & $\begin{array}{l}2732.16 \\
2732\end{array}$ & & $\begin{array}{l}5.4 \\
27\end{array}$ & & & $\left\{\begin{array}{l}7-7-6-5 \\
7-6\end{array}\right.$ & & $277=20$ & $\begin{array}{l}.0 .0 \\
7.3\end{array}$ \\
\hline & & $\left(\begin{array}{ll}0-2 \\
4-10\end{array}\right.$ & & 2732.09 & 2.7 & 140 & 2777.24 & $\left\{\begin{array}{l}5-1-4_{1} \\
5_{-1}\end{array}\right.$ & & 2777. 25 & 1.0 \\
\hline 67 & 2732.44 & & & & & & & $(3-1-20$ & & 2777. 32 & \\
\hline
\end{tabular}


TABLE 1. Comparison of observed and calculated spectra a-Continued

\begin{tabular}{|c|c|c|c|c|c|c|c|c|c|c|c|}
\hline \multirow{2}{*}{ Line } & \multirow{2}{*}{ Observed } & \multirow{2}{*}{ Transition } & \multicolumn{2}{|c|}{ Calculated } & \multirow{2}{*}{$\begin{array}{l}\text { Intensity } \\
\text { calculated }\end{array}$} & \multirow{2}{*}{ Line } & \multirow{2}{*}{ Observed } & \multirow{2}{*}{ Transition } & \multicolumn{2}{|c|}{ Calculated } & \multirow{2}{*}{$\begin{array}{l}\text { Intensity } \\
\text { calculated }\end{array}$} \\
\hline & & & $0,1,1$ & $1,1,0$ & & & & & $0,1,1$ & $1,1,0$ & \\
\hline & $c m^{-1}$ & & $\mathrm{~cm}^{-1}$ & $\mathrm{~cm}^{-1}$ & & & $\mathrm{~cm}^{-1}$ & & $\mathrm{~cm}^{-1}$ & $\mathrm{~cm}^{-1}$ & \\
\hline 141 & 2777.93 & $4_{1}-3_{1}$ & & 2778.07 & & 157 & 2790.75 & $5_{0}-4_{1}$ & $\begin{array}{l}2790.85 \\
2701\end{array}$ & & 2. 0 \\
\hline 142 & 2778.73 & $\left\{\begin{array}{l}6-3-5-3 \\
66_{-4}-5_{-2}\end{array}\right.$ & & $\begin{array}{l}2778.68 \\
2778.60\end{array}$ & $\begin{array}{l}2.6 \\
5.1\end{array}$ & $\begin{array}{l}158 \\
159\end{array}$ & $\begin{array}{l}2791.08 \\
2792.11\end{array}$ & $6-3-5-2$ & 2791.29 & & 5.7 \\
\hline $\begin{array}{l}143 \\
144\end{array}$ & $\begin{array}{l}2779.22 \\
2779.61\end{array}$ & $3_{1}-2_{2}$ & 2779.10 & & 3.2 & $\begin{array}{l}160 \\
161\end{array}$ & $\begin{array}{l}2793.06 \\
2793.26\end{array}$ & $\begin{array}{l}8-4-7-2 \\
8-3-7-3\end{array}$ & & $\begin{array}{l}2793.13 \\
2793.22\end{array}$ & $\begin{array}{l}2.3 \\
1.9\end{array}$ \\
\hline 145 & 2780.65 & $\left\{\begin{array}{l}5-5-4-4 \\
5-1-4-3\end{array}\right.$ & $\begin{array}{l}2780.61 \\
2780.61\end{array}$ & & 6.9 & 162 & 2793.90 & $\left\{\begin{array}{l}8-8-7-7 \\
8-7-7-6\end{array}\right.$ & $\begin{array}{l}2793.86 \\
2793.86\end{array}$ & & 7.2 \\
\hline 146 & 2781.29 & $\left\{\begin{array}{l}4-1-30 \\
8-8-7-6 \\
8\end{array}\right.$ & 2781.13 & 2781. 35 & $\begin{array}{l}4.4 \\
7.0\end{array}$ & $\begin{array}{l}163 \\
164\end{array}$ & $\begin{array}{l}2794.68 \\
2795.66\end{array}$ & $\left\{\begin{array}{l}7-5-6-4 \\
-\end{array}\right.$ & 2795. 66 & & 5.6 \\
\hline 147 & 2782.26 & $\begin{array}{r}18-7-7 \\
4-2-31\end{array}$ & 2782.06 & 2781.35 & $\begin{array}{l}3.5 \\
2.3\end{array}$ & 165 & 2796.46 & $\begin{array}{c}1-4-5-3 \\
6-1-50\end{array}$ & $\begin{array}{l}2795.66 \\
2796.34\end{array}$ & & 4. 6 \\
\hline 148 & 2782.97 & $\left\{\begin{array}{l}7-5-6-3 \\
7-4-6-4\end{array}\right.$ & & $\begin{array}{l}2783.01 \\
2783.03\end{array}$ & $\begin{array}{r}.6 \\
5.3\end{array}$ & $\begin{array}{l}166 \\
167\end{array}$ & $\begin{array}{l}2797.12 \\
2797.68\end{array}$ & $6-2^{--5-1}$ & 2797.27 & & 2.3 \\
\hline $\begin{array}{l}149 \\
150\end{array}$ & $\begin{array}{l}2783.50 \\
2781.18\end{array}$ & $6-2-5_{0}$ & & 2783.54 & 2.5 & 168 & 2798.10 & $\left\{\begin{array}{l}9-9-8-8 \\
9-8-8-7\end{array}\right.$ & $\begin{array}{l}2798.12 \\
2798.12\end{array}$ & & 6.2 \\
\hline 151 & 2785.12 & $\left\{\begin{array}{l}6-5-5-4 \\
6-6-5-5 \\
6\end{array}\right.$ & $\begin{array}{l}2785.10 \\
2785.10\end{array}$ & & $\begin{array}{l}7.4 \\
1.8\end{array}$ & $\begin{array}{l}169 \\
170\end{array}$ & $\begin{array}{l}2798.44 \\
2799.64\end{array}$ & & & & \\
\hline $\begin{array}{l}152 \\
153\end{array}$ & $\begin{array}{l}2786.33 \\
2786.69\end{array}$ & $\begin{array}{r}10-1-5-1 \\
5-2-4-1 \\
5-3-4-2\end{array}$ & $\begin{array}{l}2786.21 \\
2786.50\end{array}$ & 2880.11 & $\begin{array}{l}1.8 \\
1.8 \\
3.6\end{array}$ & 171 & 2800.18 & $\left\{\begin{array}{l}8-5-7-4 \\
8_{-6}-7-5 \\
6-50\end{array}\right.$ & $\begin{array}{l}2800.04 \\
2800.04 \\
2800.08\end{array}$ & & $\begin{array}{l}5.5 \\
3.5\end{array}$ \\
\hline 154 & 2787.20 & $\left\{\begin{array}{l}8-5-7-5 \\
8-6-7-4\end{array}\right.$ & 2100.00 & $\begin{array}{l}2787.30 \\
2787\end{array}$ & 2.6 & 172 & $\begin{array}{l}2800.73 \\
2801.44\end{array}$ & & & & \\
\hline 155 & 2787.77 & & & 288.50 & & 174 & $\begin{array}{l}2801.44 \\
2801.78\end{array}$ & & & & \\
\hline 156 & 2789.54 & 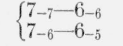 & $\begin{array}{l}2789.53 \\
2789.53\end{array}$ & & 7.4 & 175 & 2802.28 & $\left\{\begin{array}{l}10-9-9-8 \\
10-10-9-9\end{array}\right.$ & $\begin{array}{l}2802.29 \\
2802.29\end{array}$ & & 6.0 \\
\hline
\end{tabular}

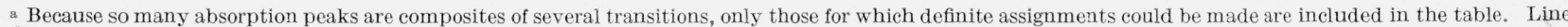
numbers correspond to numbering of peaks in figure 1

The band center of the $(0,1,1)$ band is some 40 $\mathrm{cm}^{-1}$ lower than the Bailey, Thompson, and Hale value whereas it is some $70 \mathrm{~cm}^{-1}$ higher than the center of the absorption observed by Nielsen and Nielsen.

The $A$-type band at $1906 \mathrm{~cm}^{-2}$ is in the region where one would normally expect the stretching fundamentals. The band type demands that this absorption be assigned to the $(0,0,1)$ band. Such an assignment places the band center of $\nu_{3}$ nearly $100 \mathrm{~cm}^{-1}$ lower than that reported by Nielsen and Nielsen.

\section{Discussion}

With the help of these new band centers it is possible to make some estimates of the $(0,1,0)$ band center. The difference between the $(0,1,1)$ and $(0,0,1)$ frequencies is $\sim 848 \mathrm{~cm}^{-1}$. This difference is the band center of the $(0,1,0)$ band minus $X_{23}$, the interaction between $\nu_{2}$ and $\nu_{3}$. This interaction can be estimated from the corresponding interaction in $\mathrm{H}_{2} \mathrm{~S}$ as about $10 \mathrm{~cm}^{-1}$ leading to a value of 858 $\mathrm{cm}^{-1}$ as the frequency of $(0,1,0)$. Using the $(1,1,0)$ trequency, and as $\nu_{1}$ the Raman [7] line at 1892 $\mathrm{cm}^{-1}$ one finds $851 \mathrm{~cm}^{-1}$ which includes $X_{12}$.

This frequency can also be estimated from the spectrum of crystalline [8] $\mathrm{H}_{2} \mathrm{~S}$ and $\mathrm{D}_{2} \mathrm{~S}$ which leads to a value of $855 \mathrm{~cm}^{-1}$.

The existing data in this region by Nielsen and Nielsen runs out at $853 \mathrm{~cm}^{-1}$, so this absorption seems to represent only the $R$-branch of the bending fundamental. Attempts to analyze their data as an $R$-branch were unsuccessful. Their data show no regular series that can be identified with the main strong $R$-branch series. However, the three pieces of evidence offered above strongly argue for a $\nu_{2}$ band center close to $855 \mathrm{~cm}^{-1}$ fully $80 \mathrm{~cm}^{-1}$ lower than the value previously suggested [2].

These findings argue strongly for more work on the $\mathrm{D}_{2} \mathrm{~S}$ spectrum, which will be undertaken shortly at the Bureau.

In conclusion the authors thank R. E. Florin for preparing the $\mathrm{D}_{2} \mathrm{~S}$ and Carroll Dannemiller for doing the least squares calculations on SEAC.

\section{References}

[1] C. R. Bailey, J. W. Thompson, and J. B. Hale, J. Chem. Phys. 4, 625 (1936).

[2] A. H. Nielsen and H. H. Nielsen, J. Chem. Phys. 5, 277 (1937).

[3] H. C. Allen, Jr., R. E. Naylor, and E. K. Plyler, J. Research NBS 53, 321 (1954) RP2549.

[4] E. K. Plyler, L. R. Blaine, and E. D. Tidwell, J. Research NBS 55, 279 (1955) RP2630.

[5] P. C. Cross, R. M. Hainer, and G. W. King, J. Chem. Phys. 12, 240 (1944).

[6] G. W. King, R. M. Hainer, and P. C. Cross, J. Chem. Phys. 11, 27 (1943).

[7] G. M. Murphy and J. E. Vance, J. Chem. Phys. 6, 426 (1938).

[8] J. B. Lohman, F. P. Redding, and D. F. Hornig, J. Chem. Phys. 19, 252 (1951).

Washington, March 1, 1957 